\title{
Early implant failure and physiological marginal remodeling using sandblasted and acid-etched bone level implants with $11^{\circ}$ Morse taper connection
}

\author{
Tallarico $\mathrm{M}^{1}$, Xhanari $\mathrm{E}^{2}$, Canullo $\mathrm{L}^{1}$, Meloni $\mathrm{SM}^{3}$.
}

${ }^{1}$ Private practice, Rome, Italy; ${ }^{2}$ Private practice, Tirana, Albania; ${ }^{3}$ Adjunctive prof., University of Sassari, Italy.

Introduction and aim: During the first year of function, a certain amount of physiological marginal bone loss (MBL) is expected around a dental implant; thereafter, minimal further bone loss has been observed. Several factors may increase MBL around dental implants, including surgical trauma, implant-abutment connection type, biological width establishment, mucosal tissue thickness, keratinized tissue width and bone density. The American Academy of Periodontology in 2013 defied "periimplantitis" as an inflammatory reaction associated with the loss of supporting bone beyond the initial biological bone remodeling around an implant in function, but no consensus exists with regard to define the amount of physiological bone remodeling. The objective of this study was to evaluate the implant survival and success rates as well as the physiological marginal bone remodeling expected using sandblasted and acid-etched bone level implants featuring an $11^{\circ}$ Morse taper connection within one year after loading implants.

Materials and methods: This investigation was designed as an opencohort prospective study on completely or partially edentulous patients who received at least one bone level implant with a sandblasted and acid-etched surface and an $11^{\circ}$ Morse taper connection, independently by the surgical and prosthetic protocol. Outcome measures were the success rates of the implants and prostheses, complications, marginal bone level changes, insertion torque, implant stability quotient, bone density and soft-tissue biotype.

\begin{tabular}{|c|c|c|c|c|c|c|}
\hline \multicolumn{7}{|c|}{ Implant characteristics. } \\
\hline & $7 \mathrm{~mm}$ & $8.5 \mathrm{~mm}$ & $10 \mathrm{~mm}$ & $11.5 \mathrm{~mm}$ & $13 \mathrm{~mm}$ & TOTAL \\
\hline Osstem TSIII $3.0 \mathrm{~mm}$ & - & - & - & - & 4 & 4 \\
\hline Osstem TSIII 3.5 mm & - & 2 & 6 & 27 & 10 & 45 \\
\hline Osstem TSIII $4.0 \mathrm{~mm}$ & 3 & 2 & 17 & 31 & 14 & 67 \\
\hline Osstem TSIII $4.5 \mathrm{~mm}$ & 3 & 8 & 18 & 8 & 20 & 57 \\
\hline Osstem TSIII 5 mm & - & 1 & 20 & 9 & - & 30 \\
\hline Osstem TSIII 6 mm & - & 2 & 11 & 3 & - & 16 \\
\hline Osstem TSIII 7 mm & - & 4 & 15 & 5 & - & 24 \\
\hline \multirow[t]{2}{*}{ TOTAL } & 6 & 19 & 87 & 83 & 48 & 243 \\
\hline & $\begin{array}{l}\text { Central } \\
\text { incisors }\end{array}$ & $\begin{array}{l}\text { Lateral } \\
\text { incisors }\end{array}$ & Canines & $\begin{array}{l}\text { Premolar } \\
\mathrm{s}\end{array}$ & Molars & TOTAL \\
\hline Maxilla & 26 & 7 & 4 & 45 & 41 & 123 \\
\hline Mandible & - & 15 & 5 & 42 & 58 & 120 \\
\hline TOTAL & 26 & 22 & 9 & 87 & 99 & 243 \\
\hline
\end{tabular}

Type of restorations.

\begin{tabular}{|c|c|c|c|c|c|c|}
\hline & Single & $\begin{array}{c}\text { Fixed } \\
\text { partial }\end{array}$ & $\begin{array}{c}\text { Overdenture } \\
\text { on equator }\end{array}$ & $\begin{array}{c}\text { Overdenture on } \\
\text { CAD/CAM bar }\end{array}$ & Fixed full arch & TOTAL \\
\hline Maxilla & 46 & 9 & 1 & - & 7 & 63 \\
\hline Mandible & 58 & 11 & 2 & 2 & 7 & 80 \\
\hline TOTAL & 104 & 20 & 3 & 2 & 14 & 143 \\
\hline
\end{tabular}

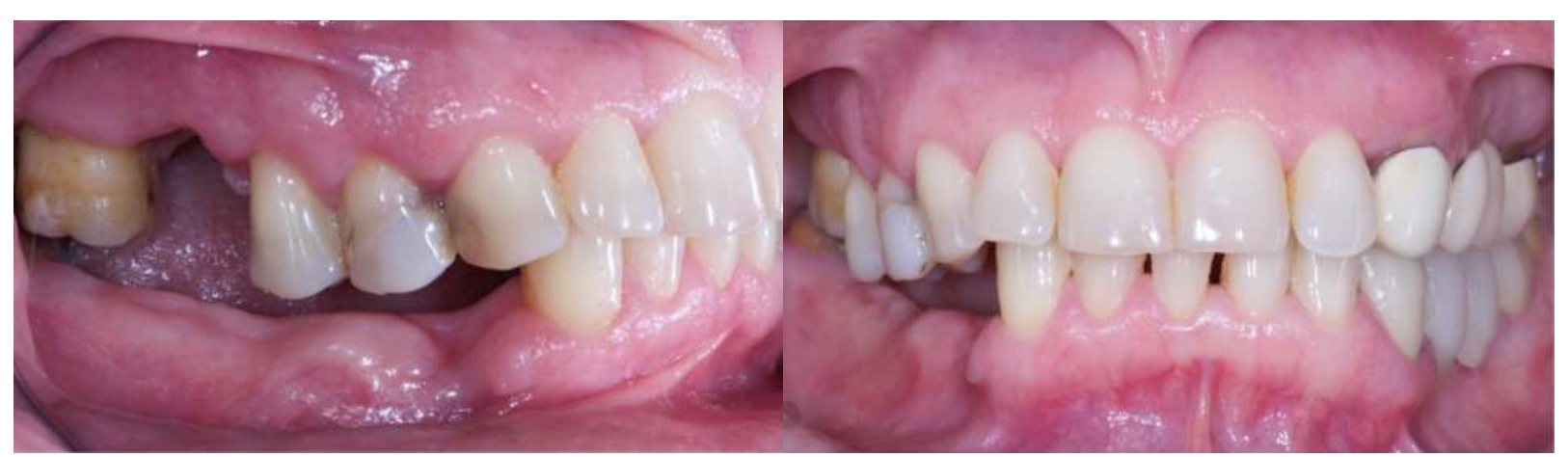

Results: A total of 243 implants were placed in 90 consecutive patients and followed up for a minimum period of one year after loading (mean of $17.6 \pm 2.5$ months; range of 12-24 months). Five implants failed in ve patients, resulting in a cumulative implant survival rate of $97.9 \%$. Insertion torque of $<35 \mathrm{~N} \mathrm{~cm}$ was found to be a risk factor for implant failure $(p=0.0068)$. No definitive prosthesis failed, resulting in a cumulative prosthetic survival rate of $100 \%$. Four patients experienced one technical complication each, resulting in a cumulative prosthetic success rate of $97.2 \%$. The cumulative mean marginal bone loss between implant placement and the follow-up one year after loading was $0.37 \pm 0.25 \mathrm{~mm}(95 \%$ CI: 0.26-0.30) (Figure 2). Comparison of marginal bone loss and the investigated risk factors found statistically higher marginal bone loss for smokers, a thin gingival biotype and guided bone regeneration $(\mathrm{p}<0.05)$.

\begin{tabular}{|c|c|c|c|c|c|c|}
\hline \multicolumn{7}{|c|}{ Marginal bone loss associated to different risks factors. } \\
\hline & & & & & & PValue \\
\hline \multirow[t]{2}{*}{ Implant location } & \multicolumn{2}{|c|}{ Maxilla (n=123) } & \multicolumn{3}{|c|}{ Mandible (n=120) } & \\
\hline & \multicolumn{2}{|c|}{$0.36 \pm 0.29$} & \multicolumn{3}{|c|}{$0.38 \pm 0.23$} & 0.7961 \\
\hline \multirow[t]{2}{*}{$\begin{array}{l}\text { Timing of } \\
\text { implant } \\
\text { placement }\end{array}$} & \multicolumn{2}{|c|}{ Postextractive (n=43) } & \multicolumn{2}{|c|}{ Delayed $(\mathrm{n}=75)$} & $\begin{array}{l}\text { Healed site } \\
\quad(\mathrm{n}=125)\end{array}$ & \\
\hline & \multicolumn{2}{|c|}{$0.36 \pm 0.29$} & \multicolumn{2}{|c|}{$0.37 \pm 0.27$} & $0.38 \pm 0.30$ & 0.9643 \\
\hline \multirow[t]{2}{*}{$\begin{array}{l}\text { Type of implant } \\
\text { placement }\end{array}$} & \multicolumn{2}{|c|}{ Guided } & \multicolumn{2}{|c|}{$\begin{array}{l}\text { Conventional free- } \\
\text { hand }\end{array}$} & & \\
\hline & \multicolumn{2}{|c|}{$0.36 \pm 0.24$} & \multicolumn{2}{|c|}{$0.37 \pm 0.26$} & & 0.8544 \\
\hline \multirow[t]{2}{*}{\begin{tabular}{|l|l|} 
Type of \\
prosthesis \\
retention
\end{tabular}} & \multicolumn{2}{|c|}{ Cemented (78) } & \multicolumn{2}{|c|}{ Screwed (165) } & & \\
\hline & \multicolumn{2}{|c|}{$0.38 \pm 0.20$} & \multicolumn{2}{|c|}{$0.36 \pm 0.23$} & & 0.5135 \\
\hline \multirow[t]{2}{*}{$\begin{array}{l}\text { Type of implant } \\
\text { loading }\end{array}$} & \multicolumn{2}{|c|}{ Immediate } & \multicolumn{2}{|c|}{ Early/conventional } & & \\
\hline & \multicolumn{2}{|c|}{$0.26 \pm 0.20$} & \multicolumn{2}{|c|}{$0.40 \pm 0.26$} & & 0.0003 \\
\hline \multirow[t]{2}{*}{$\begin{array}{l}\text { Bone } \\
\text { augmentation }\end{array}$} & $0(\mathrm{n}=172)$ & $1(\mathrm{n}=19)$ & $2(\mathrm{n}=10)$ & $3(n=3)$ & $4(n=39)$ & \\
\hline & $0.35 \pm 0.19$ & $0.51 \pm 0.50$ & $\begin{array}{l}0.22 \pm 0.1 \\
9\end{array}$ & $\begin{array}{c}0.23 \pm 0.0 \\
6\end{array}$ & $0.39 \pm 0.27$ & 0.0000 \\
\hline \multirow[t]{2}{*}{ Insertion torque } & \multicolumn{2}{|c|}{$\leq 35 \mathrm{Ncm}(\mathrm{n}=40)$} & \multicolumn{3}{|c|}{$\geq 35-45 \mathrm{Ncm}(\mathrm{n}=203)$} & \\
\hline & \multicolumn{2}{|c|}{$0.42 \pm 0.44$} & \multicolumn{3}{|c|}{$0.36 \pm 0.21$} & 0.3726 \\
\hline \multirow[t]{2}{*}{ Bone density } & \multicolumn{2}{|c|}{ Type 1 and $2(n=166)$} & & ype 3 and 2 & $(\mathrm{n}=77)$ & \\
\hline & $0.36 \pm$ & -0.23 & & $0.39 \pm 0$ & & 0.4216 \\
\hline Smoking & No smokin & $\lg (\mathrm{n}=222)$ & Smokers & $\geq 10$ sig. 1 & day $(n=21)$ & \\
\hline & $0.35 \pm$ & -0.22 & & $0.48 \pm 0$ & & 0.0098 \\
\hline $\begin{array}{l}\text { Immediate } \\
\text { abutment }\end{array}$ & Yes $(\mathrm{r}$ & n=26) & & No $(\mathrm{n}=2$ & & \\
\hline & $0.25 \pm$ & -0.19 & & $0.38 \pm 0$ & & 0.0113 \\
\hline Gingival biotype & Thin ( & $\mathrm{n}=78)$ & & Thick $(\mathrm{n}=$ & & \\
\hline & $0.43 \pm$ & $=0.27$ & & $0.34 \pm 0$ & & 0.0307 \\
\hline
\end{tabular}

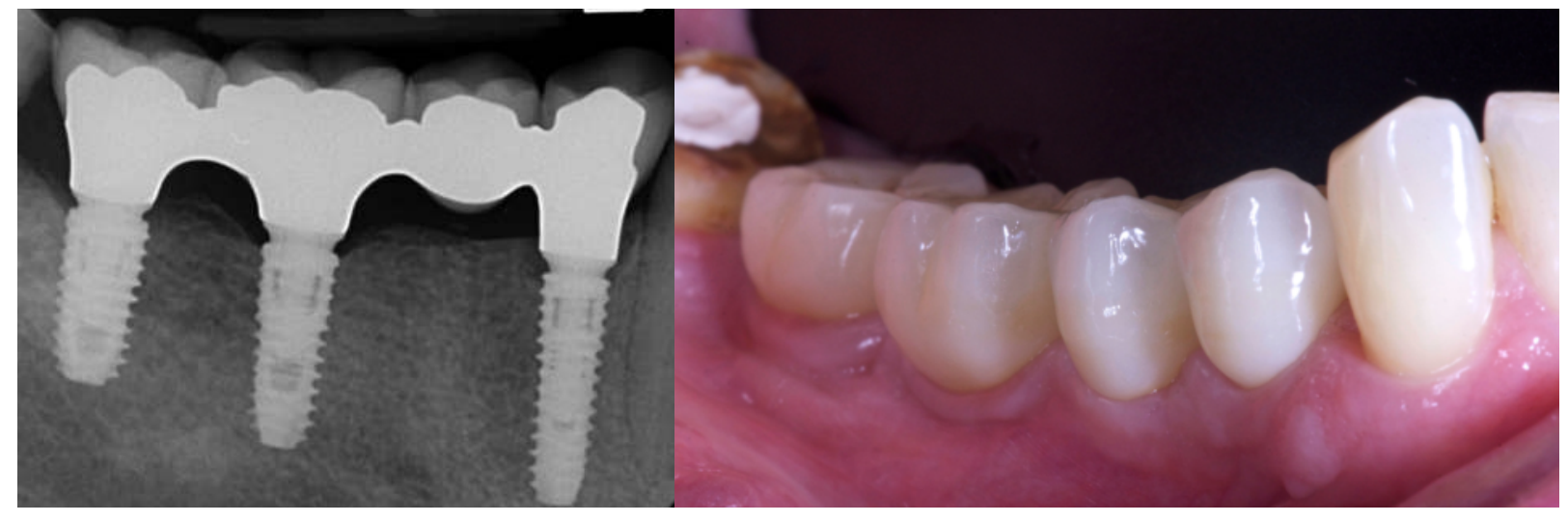

Conclusions: High implant survival/success rate and a physiological marginal bone remodeling of $0.37 \mathrm{~mm}$ can be expected using Osstem TSIII implants in the daily practice, within 1 year after loading

Galindo-Moreno P, Leon-Cano A, Ortega-Oller I, Monje A, O’Valle F, Catena A. Marginal bone loss as success criterion in implant dentistry: beyond 2 mm. 2015 Apr; 26(4):e28-34.

Canullo L, Tallarico M, Radovanovic S, Delibasic B, Covani U, Rakic M. Distinguishing predictive profiles for patient-based risk assessment and diagnostics of plaque induced, surgically and prosthetically triggered periimplantitis. 2016 Oct;27(10):1243-50. 